EPJ Web of Conferences 62, 03002 (2013)

DOI: $10.1051 /$ epjconf/20136203002

(C) Owned by the authors, published by EDP Sciences, 2013

\title{
Modelling with uncertainties: The role of the fission barrier
}

\author{
Hongliang L $\ddot{u ̈}^{1,2, a}$ and David Boilley ${ }^{1,2, b}$ \\ ${ }^{1}$ Grand Accélérateur National d'Ions Lourds (GANIL), CEA/DSM-CNRS/IN2P3, \\ Bvd. Henri Becquerel, 14076 Caen, France \\ ${ }^{2}$ Normandie Université, Caen, France
}

\begin{abstract}
Fission is the dominant decay channel of super-heavy elements formed in heavy ions collisions. The probability of synthesizing heavy or super-heavy nuclei in fusion-evaporation reactions is then very sensitive to the height of their fission barriers. This contribution will firstly address the influence of theoretical uncertainty on excitation functions. Our second aim is to investigate the inverse problem, i.e., what information about the fission barriers can be extracted from excitation functions? For this purpose, Bayesian methods have been used with a simplified toy model.
\end{abstract}

\section{Introduction}

Fission is the dominant decay channel of super-heavy elements formed by heavy ions collisions. The residue cross sections obtained in the experiments are extremely sensitive to the fission width. The fission barrier which is an essential characteristic of these nuclei cannot be directly measured and theoretical predictions can differ by few MeV. For super-heavy elements, the fission barrier is mainly due to the shell correction energy as the LDM barrier vanishes. It is then an important parameter to understand the structure of such very exotic nuclei.

Determination of 'experimental' fission barrier is then model dependent. Unfortunately, canonical models are not available yet. The fusion process to synthesize super-heavy elements is hindered with respect to what is expected from a simple extrapolation of fusion mechanisms of lighter nuclei. This socalled fusion hindrance is due to the strong Coulomb repulsion and is qualitatively well understood. But many experimental and theoretical ambiguities remain on the amplitude of the phenomenon. At contact the colliding heavy ions still face a repulsive force and have to climb a potential barrier in a dissipative environment. Models differ on the size of this inner barrier and on the strength of the friction force. As a consequence fusion probabilities can differ by orders of magnitude [1]. In this contribution we will focus on heavy nuclei for which we assumed that the fusion process is not hindered.

To investigate the determination of fission barriers from experimental data one first needs to determine uncertainties that come from both parameters and approximations of the model. In a second

\footnotetext{
ae-mail: lu@ganil.fr

be-mail: boilley@ganil.fr
}

This is an Open Access article distributed under the terms of the Creative Commons Attribution License 2.0, which permits unrestricted use, distribution, and reproduction in any medium, provided the original work is properly cited. 

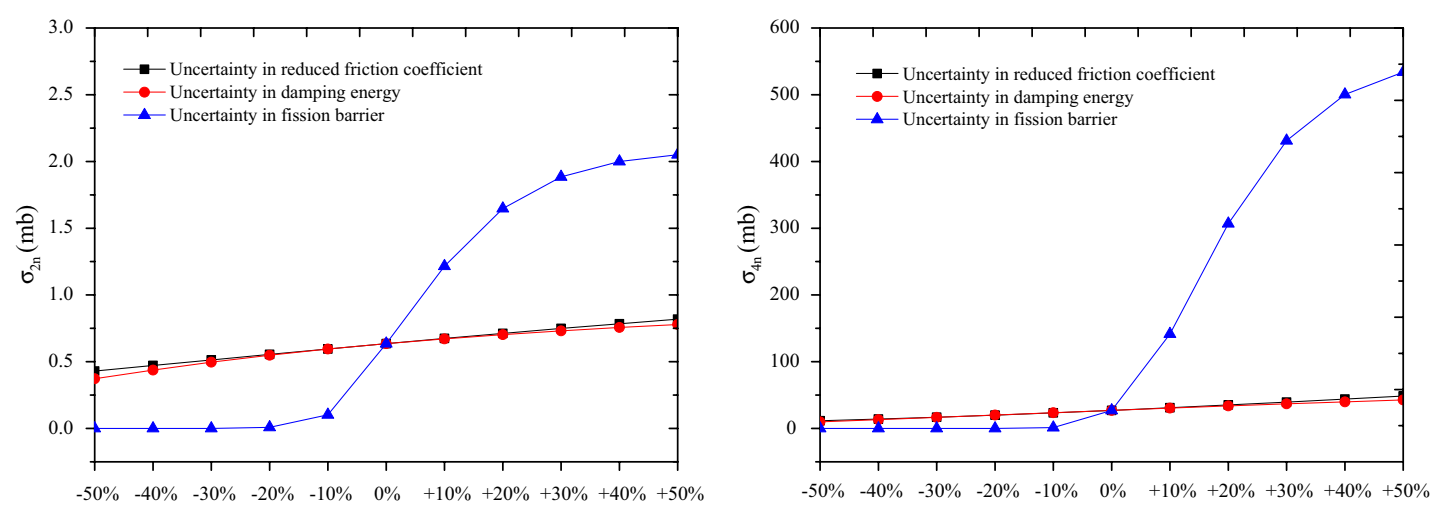

Figure 1. Effect of the variation of the three studied parameters on the maximum value of the evaporation residue cross section of the reaction ${ }^{208} \mathrm{~Pb}\left({ }^{16} \mathrm{O}, x \mathrm{n}\right){ }^{224-x} \mathrm{Th} .2 \mathrm{n}$ (left) and $4 \mathrm{n}$ (right) cases are shown.

step, we will address the inverse problem using Bayesian methods with a simplified toy model. These are preliminary results that are part of H.L.'s PhD work.

\section{Modeling with uncertainties}

\subsection{Kewpie2 code}

The Kewpie2 [2,3] code is a statistical code to study the decay of hot nuclei. It has been parametrized to study heavy and super-heavy elements and avoids Monte-Carlo schemes to quickly evaluate very rare events. The fission width is described by the Bohr and Wheeler model with Kramers and Strutinsky factors. The evaporation of light particles is described by the Weisskopf formula. See [3] for details. In this paper we will only consider two decay channels: fission and neutron evaporation.

Heavy and super-heavy elements have a small fission barrier and excited nuclei mainly decay through the fission channel. If one tunes a little the fission width, the fission probability will remain close to one. On the other hand, the residue cross sections will dramatically change. This is why we will mainly focus on the parameters and approximations of the fission width. On the other hand, such observables should be useful to constrain the fission parameters.

\subsection{Uncertainties on the parameters}

As a first step, we only consider parameters that have a large uncertainty. The main one is the fission barrier, $B_{f}$, with values varying over $1-2 \mathrm{MeV}$ range depending on the table. The evolution of the shell correction energy with the excitation energy that is usually taken into account with Ignyatuk's prescription involve a energy damping parameter, $E_{d}$, that ranges between 16 and $20 \mathrm{MeV}$. The reduced friction parameter, $\beta$, has a very large uncertainty, usual values ranging from 1 to $5 \times 10^{21} \mathrm{~s}^{-1}$. Various parameterisations of the level density are available with divergences on the behavior at low excitation energy. This problem is not addressed in the present work.

Impact of the variation with these parameters on the residue cross sections of ${ }^{208} \mathrm{~Pb}\left({ }^{16} \mathrm{O}, x \mathrm{n}\right){ }^{224-x} \mathrm{Th}$ is shown in Figure 1. It appears that the fission barrier have the strongest influence on this observable even if other parameters have a large uncertainty. 


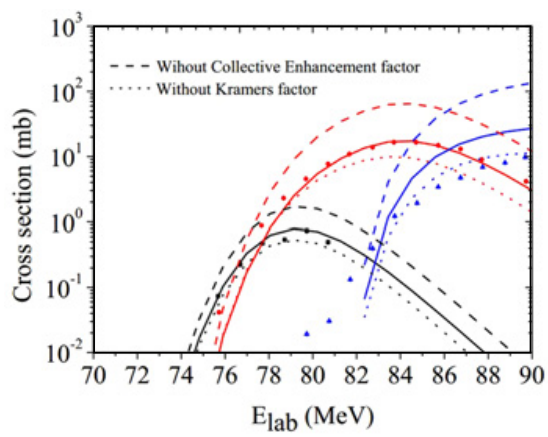

Figure 2. $2 \mathrm{n}, 3 \mathrm{n}$ and $4 \mathrm{n}$ fusion-evaporation cross-sections of the ${ }^{208} \mathrm{~Pb}\left({ }^{16} \mathrm{O}, x \mathrm{n}\right){ }^{224-x} \mathrm{Th}$ reaction as a function of energy. The solid curve corresponds to the reference model with Strutinsky-Kramers and collective enhancement factor. Dotted and dashed curves correspond to the same calculation without Kramers-Strutinsky factor or the collective enhancement factor, respectively. Data from Ref. [6] are also indicated.

\subsection{Uncertainties due to the approximations}

The various codes do not only differ by the choice of the parameters but also by the approximations or simplifications. Some take into account Kramers-Strutinsky factors [4], some others don't. Similarly with the collective enhancement factor [5] of the level density parameter. Approximations of the model lead to another contribution to the uncertainties. We will consider here two examples related to the fission width that is the most sensitive quantity.

The reference curve of Figure 2 corresponds to the full model with both Kramers-Strutinsky and collective enhancement factors and the other curves to the same calculation without one of these factors. Excitation functions of the ${ }^{208} \mathrm{~Pb}\left({ }^{16} \mathrm{O}, x \mathrm{n}\right){ }^{224-x} \mathrm{Th}$ reaction are significantly affected by these factors.

To fit the same data without Kramers-Struntisky factor, one has to increase the fission barrier by 5 to $6 \%$. And without the collective enhancement factor, one has to decrease the fission barrier by 11 to $12 \%$.

This simple calculation shows how model dependent are the so-called "experimental fission barrier".

\section{Inverse problem}

Fission barrier is the most sensitive parameter. How accurately can it be constrained by experimental data? To answer to this question, we have to solve the inverse problem to evaluate how experimental uncertainties affect the determination of the parameters.

In the previous sections we studied the influence of the uncertainty to parameters on the experimental data. Here we want to evaluated the influence of the uncertainty to the experimental data on the parameters. These two are related by Bayes theorem published exactly 250 years ago and rediscovered and first applied to physics by Laplace.

\subsection{A simple toy model with a single parameter}

Our calculations are still preliminary. We will therefore focus on the methodology and invent experimental data in order to know the true value of the fission barrier. We restrict our study to the cold fusion with evaporation of a single neutron. Residue cross section reads

$$
\sigma\left(B_{f}, E_{i}\right)=\sigma_{\text {fus }} \cdot P_{x n} \cdot \frac{\Gamma_{n}}{\Gamma_{f}+\Gamma_{n}}=\sigma_{\text {fus }} \cdot P_{\text {suvr }} .
$$


Table 1. Mean fission barrier and standard deviation determined by Bayesian inference. Various experimental situations are considered. The true value of $B_{f}=4 \mathrm{MeV}$.

\begin{tabular}{c|c|c|c|c}
\hline \hline Number of data points & Experimental uncertainty & $\left\langle B_{f}\right\rangle(\mathrm{MeV})$ & $u\left(B_{f}\right)(\mathrm{MeV})$ & $u\left(B_{f}\right) /\left\langle B_{f}\right\rangle$ \\
\hline 3 & $10 \%$ & 3.997 & 0.034 & $0.86 \%$ \\
3 & $30 \%$ & 3.968 & 0.114 & $2.87 \%$ \\
3 & $50 \%$ & 3.836 & 0.424 & $11.06 \%$ \\
6 & $50 \%$ & 3.953 & 0.145 & $3.68 \%$ \\
9 & $50 \%$ & 3.972 & 0.100 & $2.51 \%$ \\
\hline \hline
\end{tabular}

Here we will assume that the fusion cross section, $\sigma_{\text {fus }}$ is exactly known and that $P_{\text {suvr }}^{\text {exp }}$ can be directly determined from the experiments.

If the fission barrier $B_{f}$ is supposed to be the only parameter, its probability density function is given by the Bayes theorem,

$$
P\left(B_{f} \mid P_{\text {suvr }}^{\exp }, I\right)=\frac{P\left(B_{f} \mid I\right) \times P\left(P_{\text {suvr }}^{\exp } \mid B_{f}, I\right)}{\int P\left(B_{f} \mid I\right) \times P\left(P_{\text {suvr }}^{\exp } \mid B_{f}, I\right) \mathrm{d} B_{f}} .
$$

Here, $I$ stands for the hypothesis and the other parameters.

We also assume that experimental data are not correlated and are Gaussian-distributed. As a consequence,

$$
P\left(P_{\mathrm{suvr}}^{\exp } \mid B_{f}, I\right) \propto \exp \left\{-\frac{\chi^{2}}{2}\right\} \quad \text { with } \quad \chi^{2}=\sum_{i} \frac{\left(P_{\mathrm{suvr}_{i}}^{\exp }-P_{\mathrm{suvr}}\left(B_{f}, E_{i}\right)\right)^{2}}{u_{i}^{2}} .
$$

Here $u_{i}$ stands for the uncertainty to the experimental survival probability. We assume that there is no uncertainty to the energy. As we have no idea of the value of $B_{f}$, we choose an non-informative prior with a uniform distribution.

The probability density function, eq. (2), gives access to the mean value and variance of the fission barrier. Results are given in the Table 1.

The relative uncertainty to the fission barrier is lower than the one to the experimental data because the model is very sensitive to the fission barrier. As it is well known, it is also very sensitive to the experimental precision and to the number of data points.

\subsection{Correlation between experimental data}

In the previous calculation we assumed that the experimental data were independent. This should not the case because they are usually determined with the same experimental setup. But none of the experimental articles provide a covariance or a correlation coefficient. As a test, we will consider here only two data points with an arbitrary correlation coefficient $\rho$. The chi squared should then be replaced by

$$
\begin{aligned}
\chi^{2}= & \frac{1}{1-\rho^{2}}\left[\frac{\left(P_{\mathrm{suvr}_{1}}^{\exp }-P_{\mathrm{suvr}}\left(B_{f}, E_{1}\right)\right)^{2}}{u_{1}^{2}}+\frac{\left(P_{\mathrm{suvr}_{2}}^{\exp }-P_{\mathrm{suvr}}\left(B_{f}, E_{2}\right)\right)^{2}}{u_{2}^{2}}\right. \\
& \left.-\frac{2 \rho\left(P_{\text {suvr }_{1}}^{\exp }-P_{\mathrm{suvr}_{1}}\left(B_{f}, E_{1}\right)\right)\left(P_{\mathrm{suvr}_{2}}^{\exp }-P_{\mathrm{suvr}}\left(B_{f}, E_{2}\right)\right)}{u_{1} u_{2}}\right] .
\end{aligned}
$$

Results are given in Table 2.

Effect is important, but as we will see, this is probably not the most important. It would be worth evaluating the correlation coefficients when publishing experimental data. 
Table 2. Influence of the correlation coefficient on the fission barrier. The experimental precision is $50 \%$ and the number of data points 2 .

\begin{tabular}{c|c|c|c}
\hline \hline Correlation coefficient & $\left\langle B_{f}\right\rangle(\mathrm{MeV})$ & $u\left(B_{f}\right)(\mathrm{MeV})$ & $u\left(B_{f}\right) /\left\langle B_{f}\right\rangle$ \\
\hline-0.2 & 3.789 & 0.585 & $15.44 \%$ \\
-0.1 & 3.699 & 0.701 & $18.96 \%$ \\
0.0 & 3.604 & 0.801 & $22.23 \%$ \\
0.1 & 3.509 & 0.884 & $25.19 \%$ \\
0.2 & 3.417 & 0.950 & $27.78 \%$ \\
\hline \hline
\end{tabular}

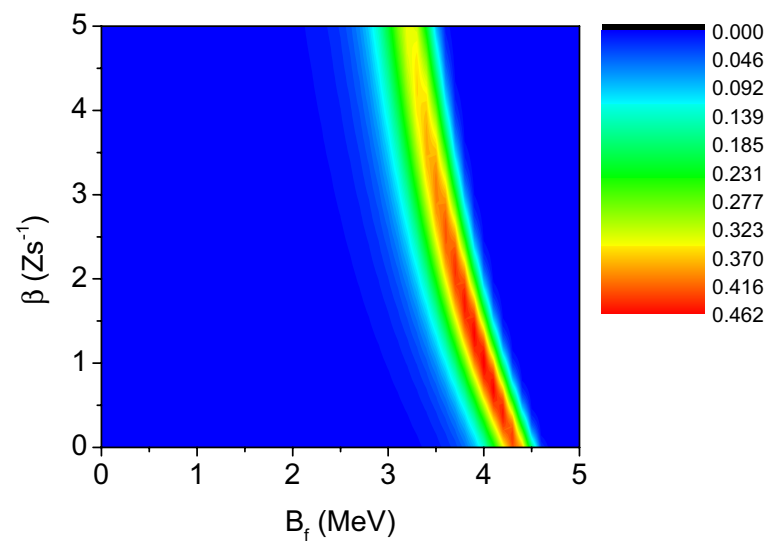

Figure 3. Probability density function of $B_{f}$ and $\beta$. The true value of $B_{f}=4 \mathrm{MeV}$ and of $\beta=2 \times 10^{21} \mathrm{~s}^{-1}$. The number of data points is fixed to 3 and the experimental precision is $50 \%$.

\subsection{Nuisance parameters}

Of course, the fission barrier is not the single parameter of the model. Other parameters with large uncertainties can also affect the final distribution of $B_{f}$. As a first test, we will only consider a single nuisance parameter here, the reduced friction coefficient, $\beta$.

With a non informative prior Bayes relation reads

$$
P\left(B_{f}, \beta \mid P_{\text {suvr }_{i}}^{\exp }, E_{i}, u_{i}\right)=\frac{P\left(P_{\text {suvr }_{i}}^{\exp } \mid B_{f}, \beta, E_{i}, u_{i}\right)}{\int P\left(P_{\text {suvr }_{i}}^{\exp } \mid B_{f}, \beta, E_{i}, u_{i}\right) d B_{f} d \beta} .
$$

In Figure 3 we plot the 2D-probability density function for these two parameters.

As expected, one cannot constrain the value of $\beta$ with this kind of observable. But, the value of the "experimental fission barrier" depends on the choice of the reduced friction parameter.

We can get ride of the nuisance parameter by marginalization,

$$
P\left(B_{f}, \mid P_{\text {suvr }_{i}}^{\exp }, E_{i}, u_{i}\right)=\frac{\int P\left(P_{\mathrm{suvr}_{i}}^{\exp } \mid B_{f}, \beta, E_{i}, u_{i}\right) d \beta}{\int P\left(P_{\text {suvr }_{i}}^{\exp } \mid B_{f}, \beta, E_{i}, u_{i}\right) d B_{f} d \beta} .
$$

and evaluate again the mean value of the fission barrier and its standard deviation. This naturally leads to an enlargement of the uncertainty.

For example with precision of $50 \%$ on three data points, one gets $\langle B f\rangle=(3.77 \pm 0,47) \mathrm{MeV}$. To get this value, we assumed that the reduced friction parameter has a uniform distribution between $1 \times 10^{21} \mathrm{~s}^{-1}$ and $5 \times 10^{21} \mathrm{~s}^{-1}$. This should be compared to the result given in Table 1 .

With a better precision on the experiential data the effect of the nuisance parameter is larger. With a precision of $10 \%$ on experimental data, one gets $B_{f}=(3.96 \pm 0.16) \mathrm{MeV}$. The uncertainty is 
almost 5 times larger when one includes the nuisance parameter. Other nuisance parameters will add a contribution to the uncertainty.

\section{Conclusion}

Uncertainties should be taken into account in modeling. How to compare or assess the prediction of model without uncertainties? How to evaluate the role of approximations?

In this contribution, we addressed the problem for some simple situations. We have presented preliminary work on the amplitude of the uncertainty to relevant parameters due to experimental data. In the case of the fusion-evaporation cross section of heavy elements we showed that the fission barrier is the parameter that has the largest influence.

To constrain this parameter with experimental data one can use Bayesian inference. If the fission barrier is considered as a single parameter, its precision is smaller that experimental precision. Unfortunately, uncertainties to nuisance parameters can be very large and significantly affect the uncertainty to the fission barrier.

These calculations are based on a simplified model and only consider 1n evaporation cross sections. Application to hot fusion reactions with evaporation of several neutrons will be more complicated.

\section{References}

[1] R. S. Naik et al, Phys. Rev. C76, 054604 (2007)

[2] B. Bouriquet et al, Comp. Phys. Com. 159, 1 (2004)

[3] A. Marchix, PhD thesis, Université de Caen Basse-Normandie (2007), http://tel.archivesouvertes.fr/tel-00197012

[4] H. A. Kramers, Physica 7, 284 (1940); V.M. Strutinsky, Phys. Lett. B47, 121 (1973)

[5] A.R. Junghans et al, Nucl. Phys. A629, 635 (1998); V. I. Zagrebaev et al, Phys. Rev. C65, 014607 (2001)

[6] C. R. Morton et al, Phys. Rev. C52, 243 (1995) 